\title{
Comparison of Surgical Outcomes of the Posterior and Combined Approaches for Repair of Cervical Fractures in Ankylosing Spondylitis
}

\author{
Panya Luksanapruksa ${ }^{1}$, Paul William Millhouse ${ }^{2}$, Victor Carlson ${ }^{2}$, \\ Thanase Ariyawatkul ${ }^{1}$, Joshua Heller ${ }^{3}$, Christopher Keppel Kepler ${ }^{2}$ \\ ${ }^{1}$ Department of Orthopedic Surgery, Faculty of Medicine Siriraj Hospital, Mahidol University, Bangkok, Thailand \\ ${ }^{2}$ Department of Orthopedic Surgery, Thomas Jefferson University \& Rothman Institute, Philadelphia, PA, USA \\ ${ }^{3}$ Department of Neurosurgery, Thomas Jefferson University, Philadelphia, PA, USA
}

\section{Study Design: Retrospective cohort study.}

Purpose: To evaluate surgical outcomes and complications of cervical spine fractures in ankylosing spondylitis (CAS) patients who were treated using either the posterior $(\mathrm{P})$ or combined approach (C).

Overview of Literature: Ankylosing spondylitis typically causes progressive spinal stiffness that makes patients susceptible to spinal fractures. CAS is a highly unstable condition. There is contradictory evidence regarding which treatment option, the posterior or the combined approach, yields superior clinical results.

Methods: A single institution database was reviewed for data in the period 1999 to 2015. All CAS patients who underwent posterior or combined instrumented fusion were enrolled. We analyzed demographic data, radiographic results, perioperative complications, and postoperative results.

Results: Thirty-three patients were enrolled (23 in the P group, 10 in the $C$ group). All patients presented with neck pain after a fall. In the P group, mean operative time was 161.1 minutes (100-327 minutes), and mean estimated blood loss (EBL) was 306.4 mL (50-750 $\mathrm{mL}$ ). In the $\mathrm{C}$ group, $90 \%$ of patients underwent a staged procedure, typically with posterior surgery first. Mean EBL was $124 \mathrm{~mL}$ (25-337 $\mathrm{mL}$ ). For posterior surgery, mean EBL was $458.3 \mathrm{~mL}(400-550 \mathrm{~mL})$. EBL of posterior surgery in the $\mathrm{C}$ group was higher but this difference was not significant $(p=0.16)$. Postoperative complication rate was higher in the $C$ group but this difference was not significant $(50 \%$ vs. $17.4 \%, p=0.09$ ). In the follow-up period, no late reoperations were performed. Patients who underwent $\mathrm{C}$ surgery had a higher rate of neurological improvement but this difference was not significant $(p=0.57)$.

Conclusions: Both $\mathrm{P}$ and $\mathrm{C}$ provided good clinical results. P surgery had lower EBL, lower postoperative complication rate, and shorter length of stay than $\mathrm{C}$ surgery; none of these differences were statistically significant.

Keywords: Cervical vertebrae; Spinal fractures; Surgery; Reoperation; Ankylosing spondylitis; Complication

Received Aug 3, 2018; Revised Oct 28, 2018; Accepted Nov 5, 2018

Corresponding author: Panya Luksanapruksa

Department of Orthopedic Surgery, Faculty of Medicine Siriraj Hospital, Mahidol University, 2 Wanglang Road, Bangkoknoi, Bangkok 10700, Thailand

Tel: +66-2-419-7969, Fax: +66-2-419-7961, E-mail: panya.luk@mahidol.ac.th 


\section{Introduction}

Ankylosing spondylitis (AS) is a chronic inflammatory spondyloarthropathy with the potential for progressive spinal stiffness which ultimately makes patients susceptible to spinal fractures, even from low-energy traumas. Cervical spine fractures in AS (CAS) is a highly unstable condition frequently leading to secondary neurological deficits $[1,2]$. Because conservative treatment has been correlated with high mortality [1], the current treatment of choice is surgical management, including decompression, fracture reduction, and instrumented fusion. Although a variety of surgical techniques has been described, the best procedure to manage patients with CAS is controversial, with options including the anterior approach (anterior cervical plate) [3,4], posterior approach (lateral mass plating and interspinous wiring of autologous rib graft) [5], lateral mass screws [6], cervical pedicle screws [7], and multilevel posterior stabilization with lateral mass or pedicle screw fixation [8], as well as combinations of the anterior and posterior approaches [1,9-12].

There is contradictory evidence regarding which treatment options yield superior clinical results in any given situation. To the best of our knowledge, there have been few studies regarding postoperative surgical outcomes for CAS. This study aimed to compare clinical outcomes (including perioperative complications, reoperation rate, and mortality) for AS patients who present with cervical fractures and who are treated with the posterior approach or combined approach.

\section{Materials and Methods}

After approval of the Thomas Jefferson University institutional review board was obtained (IRB approval no., 15D.592), we reviewed our institutional database for patients who underwent surgery for CAS between 1999 and 2015. Inclusion criteria were traumatic cervical fracture treated with posterior or combined approaches. Exclusion criteria were revision surgery, non-traumatic condition, or nonunion condition.

The patients' position was prone for the $\mathrm{P}$ group (group treated with posterior approach). A midline incision was made and paraspinal muscles were dissected. Lateral mass screws were inserted in both sides and decompressive laminectomy was performed if necessary. Posterior or posterolateral fusion was performed using either local or iliac crest autograft. The reduction techniques, number of decompressive levels, and number of fusion levels were determined by the operating surgeon. Anterior fixations were performed after posterior fixations in simultaneous or staged surgery. Patients were placed in the supine posture, after which the Smith-Peterson approach was used. The choice of anterior surgery, including bone resection, grafting techniques, bone fusion number, reconstruction approach, and the instrumentation used were also determined by the operating surgeon. Anterior cervical plates were placed one level above and below the lesion. However, if more secured fixation was needed, instrumentation was extended to two levels above and below the lesion. The choice of approach depended on the surgeon's preference, in accordance with the relative difficulty of stability and anterior approach.

Data collected through chart abstraction included demographic data: sex, age at operation, comorbidities, initial treatment, preoperative neurological status (using American Spinal Cord Injury Association [ASIA] grading), level of injury, approach, procedure details, type of implant, estimated blood loss (EBL), operative time, intraoperative complications, graft type, radiographic findings, postoperative complications, hospital length of stay (LOS), discharge status, neurological outcomes, reoperation, and mortality. Patients were also contacted via email and/ or telephone to increase follow-up rates and follow-up length, as well as to obtain additional data.

Descriptive statistics were calculated, including means, standard deviations, and ranges for quantitative data, as well as frequencies for categorical and ordinal variables. Comparative statistics used Mann-Whitney $U$-tests for continuous variables and chi-square tests for categorical variables. Statistical significance was assumed for $p$-values of $<0.05$, and odds ratios were calculated with $95 \%$ confidence intervals.

\section{Results}

Thirty-three patients were enrolled in this study. There were 31 males and two females. Twenty-three patients underwent surgery using the posterior approach and 10 patients underwent surgery using the combined approach. The most common levels of injury were to the vertebrae C6-7, C4-5, and C5-6. Most patients were placed in a halo vest prior to surgery for temporary stabilization. Demographic characteristics between groups were not 
Table 1. Demographic data and results

\begin{tabular}{|c|c|c|c|}
\hline Characteristic & Posterior approach (n=23) & Combined approach $(\mathrm{n}=10)$ & $p$-value \\
\hline Age (yr) & $68.61 \pm 11.61(42-84)$ & $70.70 \pm 10.81(50-85)$ & 0.63 \\
\hline Sex (male:female) & $21: 2$ & $10: 0$ & 0.99 \\
\hline Body mass index $\left(\mathrm{kg} / \mathrm{m}^{2}\right)$ & $27.06 \pm 8.25(20.36-57.59)$ & $27.55 \pm 2.91(22.97-31.33)$ & 0.10 \\
\hline Charlson Comorbidity Index & $3.75 \pm 1.74(1-8)$ & $4.44 \pm 1.51(1-6)$ & 0.29 \\
\hline \multicolumn{4}{|l|}{ Preoperative ASIA grade } \\
\hline$E$ & 8 & 3 & \\
\hline D & 6 & 4 & \\
\hline C & 7 & 2 & \\
\hline $\mathrm{B}$ & 0 & 0 & \\
\hline A & 2 & 1 & \\
\hline \multicolumn{4}{|l|}{ Level of injury } \\
\hline C3-4 & 1 & 0 & \\
\hline C4-5 & 8 & 1 & \\
\hline C5-6 & 5 & 2 & \\
\hline C6-7 & 9 & 5 & \\
\hline $\mathrm{C} 7-\mathrm{T} 1$ & 0 & 2 & \\
\hline Intraoperative complications (dural tear) & $1(4.65)$ & $1(10.0)$ & 0.52 \\
\hline \multicolumn{4}{|l|}{ Anterior surgery detail } \\
\hline Estimated blood loss (mL) & - & $122.4 \pm 124.3(25-337)$ & \\
\hline Operative time (min) & - & $70.2 \pm 24.2(48-110)$ & \\
\hline No. of decompression (levels) & - & $1.11 \pm 0.30(1-2)$ & \\
\hline \multicolumn{4}{|l|}{ Graft type } \\
\hline Allograft & - & 7 & \\
\hline Iliac crest & - & 3 & \\
\hline No. of fusion (levels) & - & $1.11 \pm 0.31(1-2)$ & \\
\hline Simultaneous surgery:staged surgery & - & $1: 9$ & \\
\hline Staged surgery interval (day) & - & $3.56 \pm 2.30(1-7)$ & \\
\hline \multicolumn{4}{|l|}{ Posterior surgery detail } \\
\hline Estimated blood loss (mL) & $306.38 \pm 211.08(50-750)$ & $458.33 \pm 201.03(100-700)$ & 0.16 \\
\hline Operative time (min) & $161.11 \pm 63.19(100-327)$ & $213.67 \pm 96.33(126-362)$ & 0.16 \\
\hline No. of decompressive patients & $13(56.52)$ & $4(40.0)$ & 0.47 \\
\hline No. of decompression (level) & $2.67 \pm 1.23(1-5)$ & $3.75 \pm 2.06(1-6)$ & 0.22 \\
\hline No. of fusion (levels) & $5.18 \pm 1.50(3-9)$ & $6.1 \pm 1.7(4-10)$ & 0.13 \\
\hline Graft type & & & 0.40 \\
\hline Local autograft & $18(78.26)$ & $6(60.0)$ & \\
\hline Iliac crest & $5(21.74)$ & $4(40.0)$ & \\
\hline \multicolumn{4}{|l|}{ Postoperative outcome } \\
\hline Length of stay (day) & $13.09 \pm 9.39(3-32)$ & $16.60 \pm 9.02(8-32)$ & 0.09 \\
\hline $\begin{array}{l}\text { Postoperative tracheostomy and percutaneous } \\
\text { endoscopic gastrostomy }\end{array}$ & $4(17.39)$ & $2(20.0)$ & 0.99 \\
\hline Postoperative complications & 4 (17.39) & $5(50.0)$ & 0.09 \\
\hline
\end{tabular}


Table 1. Continued

\begin{tabular}{|c|c|c|c|}
\hline Characteristic & Posterior approach $(\mathrm{n}=23)$ & Combined approach $(n=10)$ & $p$-value \\
\hline Cerebrospinal fluid fistula & 1 & 0 & \\
\hline Urinary tract infection & 0 & 2 & \\
\hline Pneumonia & 0 & 3 & \\
\hline Deep vein thrombosis & 2 & 1 & \\
\hline Dysphagia & 0 & 3 & \\
\hline Atrial fibrillation & 1 & 0 & \\
\hline Respiratory failure & 1 & 0 & \\
\hline Further neurological deficit & 1 & 0 & \\
\hline Asymptomatic screw loosening & 1 & 0 & \\
\hline Superficial wound infection & 0 & 1 & \\
\hline Mortality & 1/23 (4.65) (pneumonia) & 0 & \\
\hline Follow-up periods ${ }^{\mathrm{al}}(\mathrm{mo})$ & $69.92 \pm 54.27(6.67-179.10)$ & $65.67 \pm 69.06(6.4-195.46)$ & 0.88 \\
\hline Neurological improvement (>1 ASIA grade) $)^{b)}$ & $2 / 6(33.33)$ & $3 / 5(60.0)$ & 0.57 \\
\hline Late neurological deterioration & $2 / 6(33.33)$ & $0 / 5(0)$ & 0.46 \\
\hline Reoperation & 0 & 0 & 0.99 \\
\hline
\end{tabular}

Values are presented as mean \pm standard deviation (range), number, or number (\%).

ASIA, American Spinal Cord Injury Association grade.

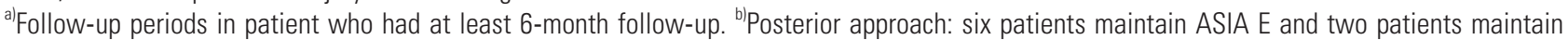
ASIA C; combined approach: one patient maintain ASIA E and two patients maintain ASIA D.

significantly different, as is shown in Table 1. Mean age at operation in the P group and $\mathrm{C}$ group (group treated with combined approach) was $68.6 \pm 11.61$ years and $70.7 \pm 10.81$ years $(p=0.63)$, respectively. All patients presented with neck pain after a fall, but of these only one-third of patients exhibited intact neurological function (11/33). However, $34.78 \%$ of patients in the P group (8/23) exhibited intact neurological function (ASIA E), as compared with $30 \%$ of patients in the $\mathrm{C}$ group. The average Charlson Comorbidity Index was $3.75 \pm 1.74$ in the P group and $4.44 \pm 1.51$ in the $C$ group $(p=0.29)$.

In the $\mathrm{P}$ group, four patients underwent decompressive surgery at an average of $2.67 \pm 1.23$ levels (range, $1-5$ levels). All patients underwent spinal fusion with lateral mass screw fixation (Fig. 1). The mean number of fused levels was 5.18 \pm 1.50 levels (range, 3-9 levels). Most patients underwent bone grafting using local autologous bone (78.26\%). Mean operative time was $162.11 \pm 63.19$ minutes (range, 100-327 minutes), and mean intraoperative blood loss was $306.38 \pm 211.08 \mathrm{~mL}$ (range, $50-750 \mathrm{~mL}$ ).

In the $\mathrm{C}$ group, $90 \%$ of the patients underwent a staged procedure, typically with the posterior surgery first. Average interval between surgeries was $3.56 \pm 2.30$ days (range, 1-7 days). All patients underwent combined spinal fusion
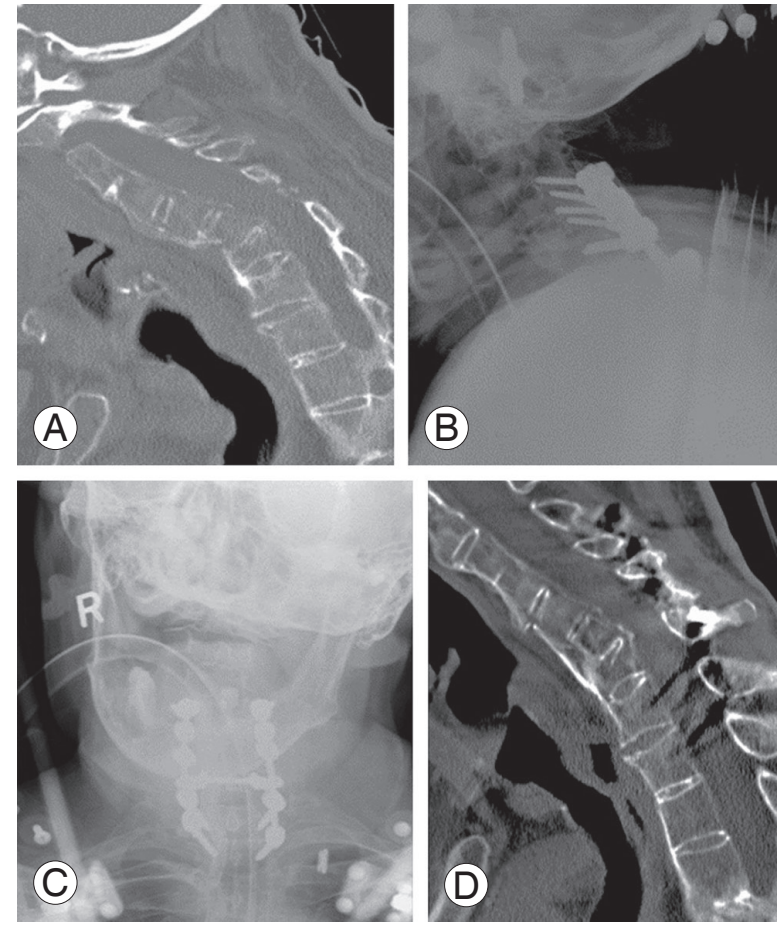

Fig. 1. Radiographic study of an 84-year-old man (patient \#7). (A) Lateral view of cervical spine CT scan demonstrates C6-7 fracture with kyphosis. (B, C) Postoperative anteroposterior and lateral radiograph demonstrates posterior cervical stabilization using lateral mass screws system. (D) Lateral view of cervical CT scan 1 year later demonstrates bone bridge across the fracture site. CT, computed tomography. 

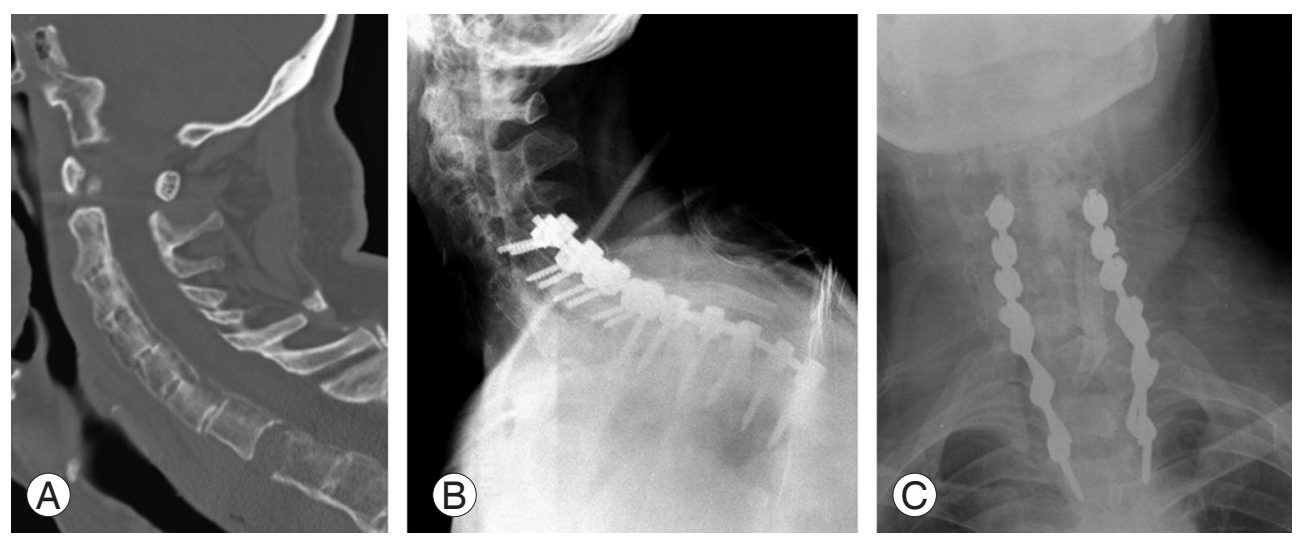

Fig. 2. Radiographic study of a 74-year-old man (patient \#17). (A) Lateral view of cervical spine computed tomography scan demonstrates $\mathrm{C7T1}$ distractive fracture. (B, C) Postoperative anteroposterior and lateral radiograph demonstrates posterior cervical stabilization using the lateral mass screws system.
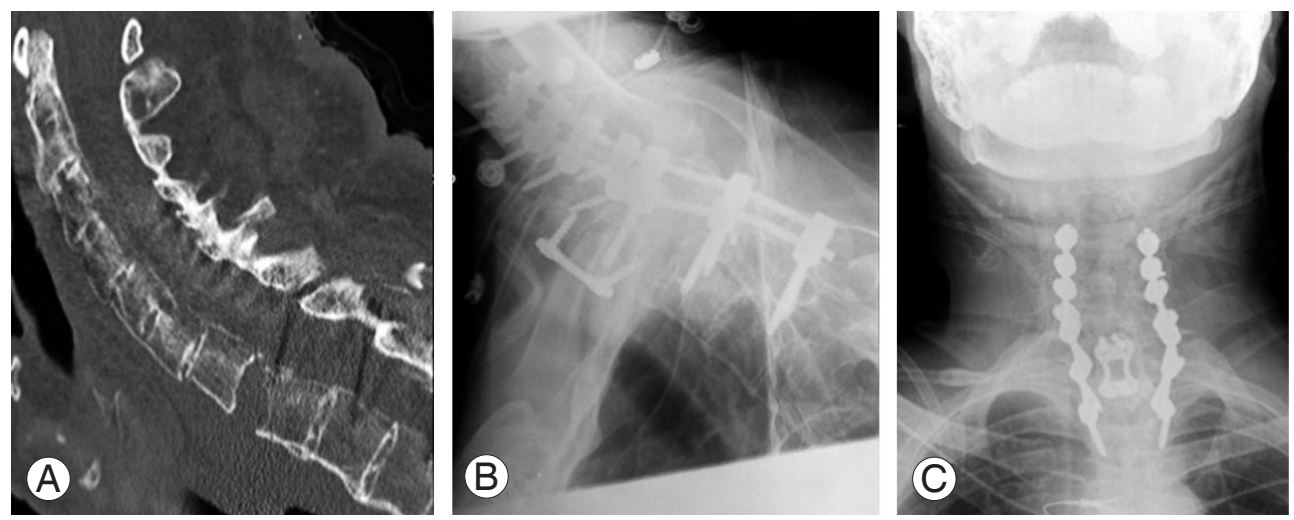

Fig. 3. Radiographic study of a 74-year-old man (patient \#17) after surgery. (A) Lateral view of cervical spine computed tomography scan demonstrates C7T1 gap after posterior fixation. (B, C) Postoperative anteroposterior and lateral radiographs demonstrate combined anterior posterior cervical stabilization using the lateral mass screws system and cervical plate.

using lateral mass screw fixation and anterior surgery (Figs. 2, 3). Anterior surgery included discectomy or corpectomy with instrumented fusion. The mean number of fused levels and mean EBL for anterior surgery were 1.11 levels and $122.4 \pm 124.28 \mathrm{~mL}$ (range, $25-337 \mathrm{~mL}$ ), respectively. Posterior surgery was performed at a mean of $6.10 \pm 1.70$ levels (range, 4-10 levels) and mean EBL was $458.33 \pm 201.03 \mathrm{~mL}$ (range, 100-700 mL). Sixty percent of patients used only local autografts to encourage fusion, while the remainder also used allograft bone in posterior surgery. There was one intraoperative dural tear in both groups. When compared with the P group, the intraoperative blood loss of posterior surgery in the $\mathrm{C}$ group was higher, but this difference was not statistically significant $(p=0.16)$. Moreover, the LOS of the $\mathrm{C}$ group was longer than was that of the P group, but this difference was also not statistically significant (16.6 \pm 9.0 versus $13.1 \pm 9.4$ days, $p=0.09$ ).

Nine patients had postoperative complications. The postoperative complication rate tended to be higher in the C group (50\% versus $17.4 \%, p=0.09$ ) than it was in the $\mathrm{P}$ group. In the $\mathrm{P}$ group, four patients had complications, including cerebrospinal fluid fistula, deep vein thrombosis (DVT), atrial fibrillation, and asymptomatic implant loosening; one patient developed further neurological deficit postoperatively from epidural hematoma, which required urgent evacuation. In the $\mathrm{C}$ group, postoperative complications occurred in five patients, and these included pneumonia, dysphagia, DVT, wound infection, and urinary tract infection. There were 18 patients with follow-up periods of longer than 6 months (12 in the P group and six in the $\mathrm{C}$ group). The mean follow-up periods for the $\mathrm{P}$ and C groups were 69.9 and 65.7 months, respectively $(p=0.88)$. In the follow-up period, no late reoperation was needed 


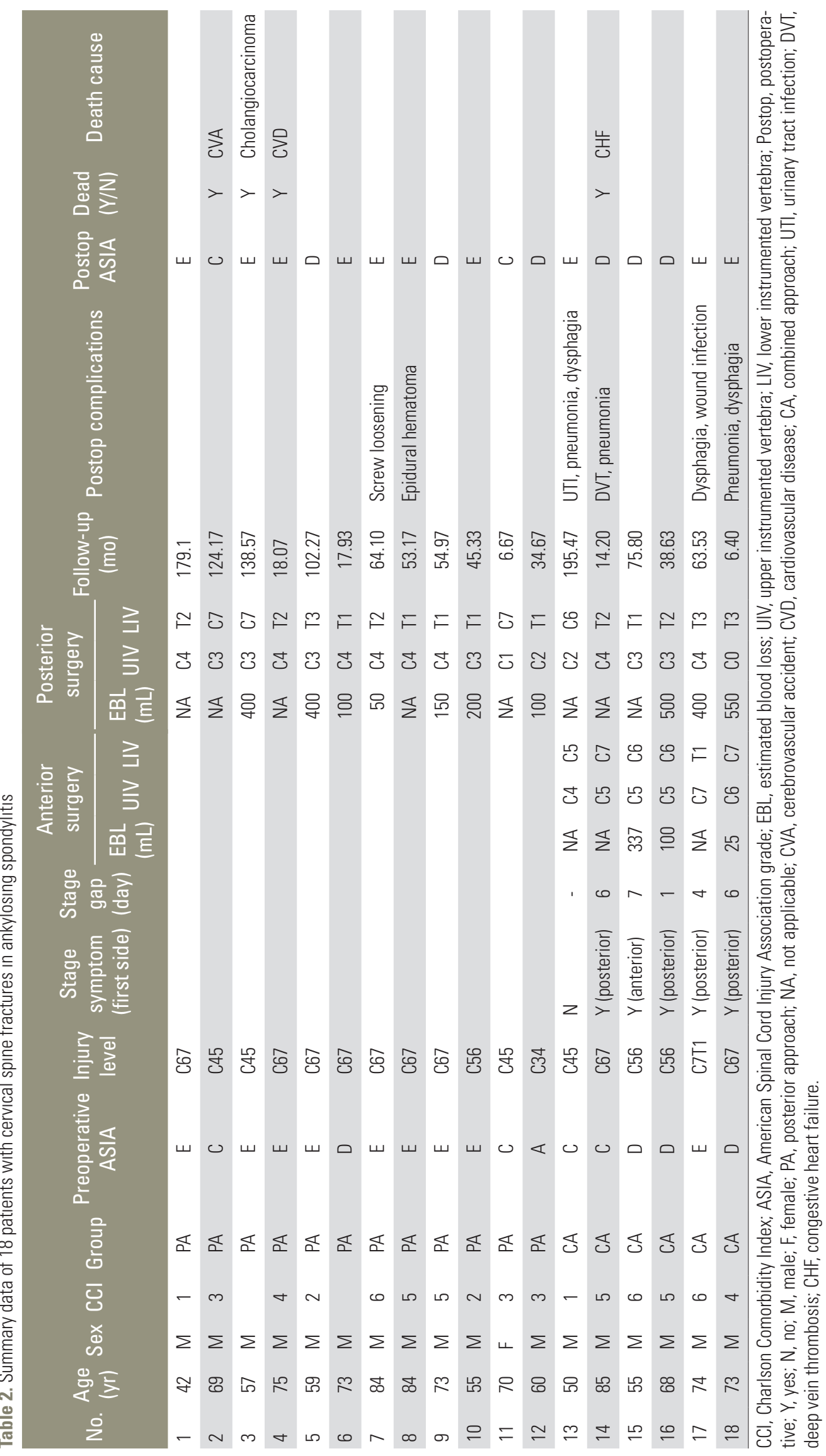


in either group, although one patient in the P group developed asymptomatic loosing of lateral mass screws. In terms of neurological outcome, patients in the $\mathrm{C}$ group who underwent surgery had a higher rate of improvement as compared with rate of improvement after surgery in the P group, but this difference was not statistically significant (60\% versus $33.3 \%, p=0.57$ ). Details for each patient are shown in Table 2.

\section{Discussion}

Spinal injury in AS patients has some unique characteristics. The spine in AS patients is rigid, appearing as a long bone with coexisting osteoporotic bone and cervical kyphosis. For these reasons, the majority of AS patients sustain low-energy injuries, which may comprise a fall from standing height. Hyperextension is the most common mechanism of injury. The fracture could occur through the vertebral body or the intervertebral disc. Transdiscal fracture and fracture through osteoporotic bone are difficult to detect and delayed diagnosis might occur. Additionally, CAS is a highly unstable condition that commonly causes progressive neurological deficit $[1,2]$. Gilard et al. [13] reported neurological decline in $4 / 7$ patients (57.14\%) who had CAS; before injury these patients lived independently, but they were unable to do so afterwards. The treatment of choice is currently surgical management with anterior, posterior, or combined approaches. There is minimal literature with regard to postoperative surgical outcomes for CAS patients. The current study showed that both posterior and combined approaches yielded good results, including improvement of neurological status without the need for later reoperation. The P group demonstrated trends suggestive of worse neurological recovery, as compared with neurological recovery in the C group (33.33\% versus $60 \%, p=0.57$ ); however, the $\mathrm{P}$ group exhibited an improved postoperative complication rate $(17.39 \%$ versus $50 \%, p=0.09)$. The $\mathrm{P}$ group had two patients with postoperative neurological deterioration, while the $\mathrm{C}$ group had none. However, the cause of late neurological deterioration was unclear, and may include progressive kyphosis. All patients achieved successful fusion. The P group had lower intraoperative blood loss and LOS, but these differences were not significant.

The best approach for treating this difficult patient population remains controversial. With regard to the posterior approach, Robinson et al. [14] in their study conducted a prospective cohort study in which they reported complications and survival after surgery in which long posterior instrumentation constructs were used to treat cervical and cervicothoracic fractures in AS patients. Of the 41 patients enrolled, 11 (26.8\%) had postoperative complications, which included five with postoperative infections, three with pneumonia, two with tracheostomy, and one with postoperative cerebrospinal fluid leakage due to accidental durotomy. No patient required reoperation due to implant failure or nonunion. They concluded that posterior instrumentation of CAS is recommended, but that complications were not unusual [14]. Taggard and Traynelis [5] in their study reported clinical outcomes of seven CAS patients who underwent lateral mass plating and interspinous wiring of an autologous rib graft with postoperative use of a cervical collar. No patient showed postoperative neurologic deterioration. Radiographic evidence of fusion was observed in the five patients available for follow-up [5]. With regard to the combined approach, Metz-Stavenhagen et al. [15] suggested that in cases of severe kyphosis, such an approach is necessary but carries a higher risk of complications. Payer [16] in their study reported treatment of four consecutive CAS cases that were treated with a combined approach. After the mean follow-up period of 11 months, all patients showed good alignment without deformity.

Longo et al. [17] in their study performed a systematic review in 2015 comparing the clinical outcomes and complication rates of anterior, posterior, and combined approaches. Eight studies with a total of 110 patients were included. Notably, they showed that cervical fractures were more common in men (94\%) and that they typically occurred in middle-aged patients (mean age, 59.2 years). The most common levels were C6-7, C5-6, and C4-5. Most patients had postoperative neurological improvement of at least 1 Frankel grade (78\% in combined approach, $79 \%$ in anterior approach, and $70 \%$ in posterior approach). All patients who underwent radiographic assessment were able to heal the index fracture. Overall, the postoperative medical complication rate was $19 \%$ (14\% in anterior approach, $26 \%$ in posterior approach, and $16 \%$ in combined approach), while the intraoperative complication rate was $16 \%$ (14\% in anterior approach, $15 \%$ in posterior approach, and $18 \%$ in combined approach). The most common intraoperative complications were loosening of implants and epidural hematoma. The most common postoperative complications were pneumonia, 
infection, and deformity. They concluded that combined and posterior approaches were more effective than was the anterior approach, which was associated with a higher risk of implant failure and subsequent revision surgery [17].

The results of the present study supported those of previous studies, including successful union in all cases without the need for reoperation. However, we found that the postoperative complication rate was higher in $\mathrm{C}$ group, but failed to reach statistical significance, possibly due to the low number of patients. Cornefjord et al. [18] in their study reported operative results in CAS. Fifteen patients were treated with long posterior fixation constructs, while four patients were treated with combined posterior fixation and anterior plate fixation. The perioperative complication rate was $26.31 \%(5 / 19)$, which included one C6 perforated pedicle, instrumentation placement errors, extensive perioperative bleeding, and deep-wound infection that required surgical drainage. However, no patients required reoperation due to implant loosening or healing problems [18]. However, the present study had more medical complications than did the Cornefjord study.

We found that patients with CAS treated using a posterior approach had shorter LOS and lower postoperative complication rate than did patients treated using a combined approach. While the fusion and reoperation rates were good in both groups, neurological recovery rate was higher in the $\mathrm{C}$ group. Therefore, we recommend posterior surgery for CAS.

The present study was a large study comparing clinical outcomes between the different approaches used in 33 patients. Most patients were operated on after the year 2000 , and received the benefits of modern instrumentation (lateral mass screw and rod system). The limitations of this study included that it was a single-center retrospective study, had a small number of participants, and exhibited a moderate dropout rate. Because some patients demonstrated intact neurological function, the benefits of neurologic decompression and recovery could not be similarly evaluated in all patients. A large, multi-center, randomized control study may allow additional evaluation of the optimal surgical approach to treat AS patients with cervical fracture.

\section{Conclusions}

CAS patients have highly unstable injuries that frequently require decompression and stabilization. Both posterior and combined approach provide good clinical results, with respect to fusion rate and the need for reoperation. The posterior surgical approach had lower EBL and postoperative complication rate, as well as a shorter LOS; however, these differences were not statistically significant.

\section{Conflict of Interest}

No potential conflict of interest relevant to this article was reported.

\section{Acknowledgments}

The authors would like to thank Miss Natnicha Sriburiruk for editing the manuscript and assisting in preparation for journal submission.

\section{References}

1. Altenbernd J, Bitu S, Lemburg S, et al. Vertebral fractures in patients with ankylosing spondylitis: a retrospective analysis of 66 patients. Rofo 2009;181:45-53.

2. Caron T, Bransford R, Nguyen Q, Agel J, Chapman J, Bellabarba C. Spine fractures in patients with ankylosing spinal disorders. Spine 2010;35:E458-64.

3. Heineck J, Bergert H, Muller M, et al. Ventral fusion of a fracture of the cervical spine in ankylosing spondylitis and struma permagna. Unfallchirurg 2007;110:571-5.

4. Kouyoumdjian P, Guerin P, Schaelderle C, Asencio $\mathrm{G}$, Gille O. Fracture of the lower cervical spine in patients with ankylosing spondylitis: retrospective study of 19 cases. Orthop Traumatol Surg Res 2012;98:54351.

5. Taggard DA, Traynelis VC. Management of cervical spinal fractures in ankylosing spondylitis with posterior fixation. Spine 2000;25:2035-9.

6. Tamburrelli FC, Rossi B, Pola MB. Vertebral epidural hematoma: pathogenesis and treatment. Eur Spine J 2012;21:813-4.

7. Ding S, Zheng K. Artificial total hip arthroplasty with collum femoris preserving for treating hip joint. Zhongguo Xiu Fu Chong Jian Wai Ke Za Zhi 2010;24:1-4.

8. Hahn P, Komp M, Merk H, Godolias G, Ruetten S. Surgical options in fractures of the cervical and 
thoracic spine on patients with ankylosing spondylitis: prospective study of 21 patients. Eur Spine J 2010;19:2040.

9. Duhem-Tonnelle V, Duhem R, Allaoui M, Chastanet $\mathrm{P}$, Assaker R. Fracture luxation of the cervical spine in patients with ankylosing spondylitis: six cases. Neurochirurgie 2008;54:46-52.

10. Einsiedel T, Schmelz A, Arand M, et al. Injuries of the cervical spine in patients with ankylosing spondylitis: experience at two trauma centers. J Neurosurg Spine 2006;5:33-45.

11. Papagelopoulos PJ, Sapkas GS, Kateros KT, Papadakis SA, Vlamis JA, Falagas ME. Halo pin intracranial penetration and epidural abscess in a patient with a previous cranioplasty: case report and review of the literature. Spine 2001;26:E463-7.

12. Xiang LB, Yu HL, Liu J, Chen Y, Yang HF. One-stage surgery by a combined anterior-posterior approach to treat ankylosing spondylitis complicated by a multiple-level cervical vertebral chance fracture. Mod Rheumatol 2015;25:282-5.

13. Gilard V, Curey S, Derrey S, Perez A, Proust F. Cer- vical spine fractures in patients with ankylosing spondylitis: importance of early management. Neurochirurgie 2014;60:239-43.

14. Robinson Y, Robinson AL, Olerud C. Complications and survival after long posterior instrumentation of cervical and cervicothoracic fractures related to ankylosing spondylitis or diffuse idiopathic skeletal hyperostosis. Spine (Phila Pa 1976) 2015;40:E227-3.

15. Metz-Stavenhagen P, Krebs S, Meier O. Cervical fractures in ankylosing spondylitis. Orthopade 2001;30:925-31.

16. Payer M. Surgical management of cervical fractures in ankylosing spondylitis using a combined posterior-anterior approach. J Clin Neurosci 2006;13:73-7.

17. Longo UG, Loppini M, Petrillo S, Berton A, Maffulli N, Denaro V. Management of cervical fractures in ankylosing spondylitis: anterior, posterior or combined approach? Br Med Bull 2015;115:57-66.

18. Cornefjord M, Alemany M, Olerud C. Posterior fixation of subaxial cervical spine fractures in patients with ankylosing spondylitis. Eur Spine J 2005;14:4018. 